\title{
Fluorine F 18 Fluoro-PEG6-IPQA
}

National Cancer Institute

\section{Source}

National Cancer Institute. Fluorine F18 Fluoro-PEG6-IPQA. NCI Thesaurus. Code C96235.

A radioconjug ate containing the tracer PEG6-IPQA labeled with fluorine $\mathrm{F} 18$ for potential tumor imaging using positron emission tomography (PET). Upon administration, the IPQA moiety of fluorine F 18 fluoro-PEG6-IPQA selectively targets and irreversibly binds to the constitutively active mutant L858R of epidermal growth factor receptor (EGFR) kinase, thereby allowing the visualization of tumor cells expressing the active mutant L858R EGFR using PET. This can be used to select EGFR kinase inhibitors that bind in a similar manner as this tracer and may allow individualized therapy for patients that respond well to these types of EGFR kinase inhibitors. The presence of the L858R EGFR mutation in non small cell lung cancer (NSCLC) cells is correlated with a better response to EGFR kinase inhibitors compared to wild-type (WT) or L858R/T 790M EGFR dualmutant. 